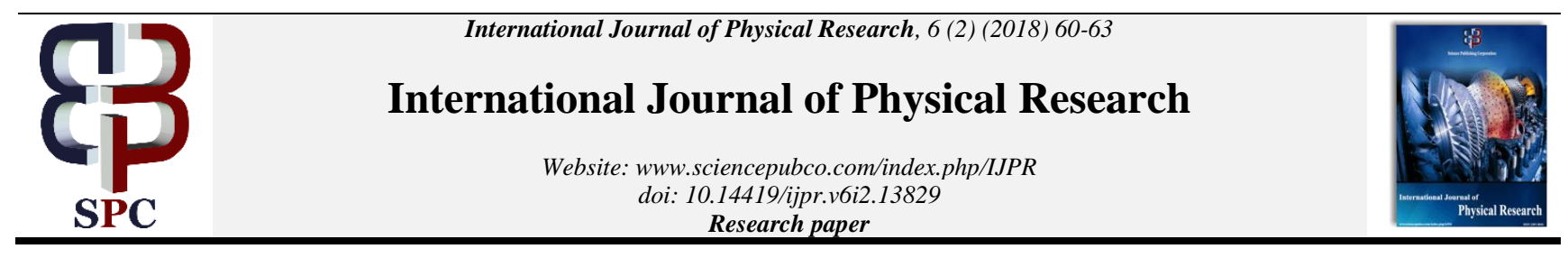

\title{
Dark and singular soliton solutions of perturbed Gerdjikov-Ivanov equation via the first integral method
}

\author{
Salam Subhaschandra Singh * \\ Physics Department, Imphal College, Imphal-795001, Manipur, India \\ *Corresponding author E-mail: subhasic@yahoo.co.in
}

\begin{abstract}
This paper employs the first integral method in obtaining dark and singular soliton solutions of perturbed Gerdjikov-Ivanov equation showing that the method is a powerful tool for finding exact solutions of many nonlinear evolution (NLE) equations which are found in the studies of social dynamics, nonlinear science and engineering.
\end{abstract}

Keywords: Fiber Optics; First Integral Method; Gerdjikov-Ivanov Equation; Group Velocity Dispersion; Solitons.

\section{Introduction}

The well-known nonlinear Schrodinger (NLS) equation is one of the most important nonlinear evolution (NLE) equations encountered in the studies of many branches of physics like quantum field theory (QFT) and fiber optics. Many extended versions of this equation with higher order nonlinearities have also been extensively studied in many areas of nonlinear physics and mathematics. Among them, mention may be made of higher order nonlinear Schrodinger (HONLS) equation and derivative nonlinear Schrodinger (DNLS) equation. In recent years, the study of soliton dynamics has become very important as the soliton technology is now-a-days extensively used in trans-continental and transoceanic communications through optical fibers and also in various social media communications like facebook and twitter. The present paper studies the dark and the singular soliton solutions of a perturbed Gerdjikov-Ivanov (GI ) equation in $(1+1)$ dimension. The GI equation describes optical soliton propagation in $(1+1)$ dimensions. This equation has been studied by many authors in recent years.

The perturbed GI equation [1 - 3] which is going to be studied in this paper has the form

$$
\begin{aligned}
& i q_{t}+a q_{x x}+b|q|^{4} q \\
& =i\left[c q^{2} q_{x}^{*}+\alpha q_{x}+\lambda\left(|q|^{2} q\right)_{x}+\mu\left(|q|^{2}\right)_{x} q\right]
\end{aligned}
$$

Here, $q(x, t)$ represents a complex-valued wave profile with $x, t$ as spatial and temporal variables respectively. The first term represents a linear temporal evolution, a is the group velocity dispersion ( GVD ) coefficient, b is the quintic nonlinearity coefficient, c is the nonlinear dispersion coefficient, $\alpha$ is the inter-modal dispersion coefficient, $\lambda$ is the self-steepening coefficient, $\mu$ is the higher order dispersion coefficient and $\mathrm{i}=\sqrt{(}(-1)$ is the imaginary number. The asterik symbol denotes complex conjugation of the mathematical quantity.
2. Reduction of a given nonlinear partial differential equation to a nonlinear ordinary differential equation

In this section, let us describe how a given nonlinear partial differential equation (NLPDE) can be reduced to a nonlinear ordinary differential equation (NLODE).

Considering a given NLPDE having the form

$\mathrm{F}\left(\mathrm{u}, \mathrm{u}_{\mathrm{t}}, \mathrm{u}_{\mathrm{x}}, \mathrm{u}_{\mathrm{tt}}, \mathrm{u}_{\mathrm{tx}}, \mathrm{u}_{\mathrm{xx}}, \ldots\right)=0$,

where $\mathrm{F}$ is a polynomial in $\mathrm{u}(\mathrm{x}, \mathrm{t})$ and its partial derivatives, let $\mathrm{us}$ introduce the transformations

$u(x, t)=U(\xi), \xi=x-v t$.

Here, $x, t$ are spatial and temporal variables respectively, $u(x, t)$ represents a wave profile and $\mathrm{v}$ is a constant (generally the constant speed of wave propagation).

Now, we have

$\mathrm{u}_{\mathrm{t}}=-\mathrm{v} \mathrm{U}^{\prime}(\xi), \mathrm{u}_{\mathrm{x}}=\mathrm{U}^{\prime}(\xi)$,

$u_{t t}=v^{2} U^{\prime \prime}(\xi), u_{t x}=-v U^{\prime \prime}(\xi)$,

$\mathrm{u}_{\mathrm{xx}}=\mathrm{U}^{\prime \prime}(\xi)$, etc.

where

$U^{\prime}(\xi)=\frac{d U(\xi)}{d \xi}, U^{\prime \prime}(\xi)=\frac{d^{2} U(\xi)}{d \xi^{2}}$, etc.

In terms of the new function $U(\xi)$ and its derivatives, Eq. (2) is transformed into another polynomial equation of the form 


$$
H\left(U(\xi), U^{\prime}(\xi), U^{\prime \prime}(\xi), U^{\prime \prime \prime}(\xi), \ldots\right)=0
$$

where $\mathrm{H}$ is a polynomial in $\mathrm{U}$ and its derivatives.

Then, we are to solve this reduced nonlinear ordinary differential equation (RNLODE).

\section{Algorithm of the first integral method}

Feng [4 - 7] had originally established the first integral method which is based on the ring theory of commutative algebra. This method has latter been applied by many authors in solving different types of nonlinear evolution (NLE) equations encountered in the studies of nonlinear science and engineering [8], [9]. The method is briefly described as in the following.

To solve a nonlinear ordinary differential equation (NLODE) by the first integral method, let us describe an algorithm of the method. The method involves the following steps.

\section{First Step:}

We assume

$\mathrm{U}(\xi)=\mathrm{X}(\xi)$

And introduce a new variable

$Y(\xi)=X^{\prime}(\xi)$

Second Step:

Under the above conditions, Eq. (4) can be converted to a plane autonomous system of NLODEs as in the following.

$\mathrm{X}^{\prime}(\xi)=\mathrm{Y}(\xi), \mathrm{Y}^{\prime}(\xi)=\mathrm{R}(\mathrm{X}(\xi), \mathrm{Y}(\xi))$

where $\mathrm{R}$ is a function of $\mathrm{X}(\xi)$ and $\mathrm{Y}(\xi)$ and the primes denote derivatives with respect to $\xi$.

If we can find two first integrals to the system of differential equations in Eq. (7) under the same conditions, then the analytic solutions of Eq. (7) can be obtained directly. However, in general, it is really difficult for us to realize this even for one first integral, because for a given plane autonomous system, there exists neither a systematic theory that can tell us how to find its first integrals nor a logical way for telling us what these first integrals are. We will apply the Division Theorem to obtain a first integral to the system of equations in (7) which reduces Eq. (4) to a first order integrable ODE. An exact solution of Eq. (2) is then obtained by solving this ODE. For convenience, we recall the Division Theorem for two variables in the complex domain $\mathrm{C}[\mathrm{w}, \mathrm{z}]$.

Division theorem:

For two polynomials $\mathrm{P}(\mathrm{w}, \mathrm{z})$ and $\mathrm{Q}(\mathrm{w}, \mathrm{z})$ in a complex domain $C[w, z]$, if $P(w, z)$ is irreducible in $C[w, z]$ and if $Q(w, z)$ vanishes at all zero points of $P(w, z)$, then there exists another polynomial $G(w, z)$ in $C[w, z]$ such that

$\mathrm{Q}(\mathrm{w}, \mathrm{z})=\mathrm{P}(\mathrm{w}, \mathrm{z}) \mathrm{G}(\mathrm{w}, \mathrm{z})$

The division theorem follows immediately from Hilbert- Nullstellensatz theorem of commutative algebra.

\section{Reduction of perturbed gerdjikov-ivanov equation to a NLODE}

In Eq. (1), let us put

$$
q(x, t)=U(\xi) e^{i \phi(x, t)}
$$

with

$$
\xi=\mathrm{x}-\mathrm{vt} \text { and } \phi(\mathrm{x}, \mathrm{t})=-\mathrm{kx}+\omega \mathrm{t}+\theta
$$

where $\mathrm{x}$ is a spatial variable, $\mathrm{t}$ is a temporal variable, $\mathrm{v}$ is the constant speed of wave propagation, $U(\xi)$ represents the pulse shape, $\phi(x, t)$ is the phase component of the wave, $k$ is frequency of the soliton, $\omega$ is the wave number and $\theta$ is a phase constant.

Now, we have

$q^{*}(\mathrm{x}, \mathrm{t})=\mathrm{U}(\xi) \mathrm{e}^{-\mathrm{i} \phi(\mathrm{x}, \mathrm{t})}$,

$i q_{t}=\left(-i v \frac{d U}{d \xi}-\omega U\right) e^{i \phi}$,

$a q_{x x}=a\left(\frac{d^{2} U}{d \xi^{2}}-2 i k \frac{d U}{d \xi}-k^{2} U\right) e^{i \phi}$

$b|q|^{4} q=b U^{5} e^{i \phi}$

$-i c q^{2} q_{x}^{*}=\left(-i c U^{2} \frac{d U}{d \xi}+c k U^{3}\right) e^{i \phi}$,

$i \alpha q_{x}=\left(i \alpha \frac{d U}{d \xi}+\alpha k U\right) e^{i \phi}$,

$i \lambda\left(|q|^{2} q\right)_{x}=\left(3 i \lambda U^{2} \frac{d U}{d \xi}+\lambda k U^{3}\right) e^{i \phi}$,

$i \mu\left(|q|^{2}\right)_{x} q=2 i \mu U^{2} \frac{d U}{d \xi} e^{i \phi}$.

Substituting these relations in Eq. (1) and then separating the real and the imaginary parts of the resulting equation, we obtain

Real Part:

$a \frac{d^{2} U}{d \xi^{2}}-\left(\omega+\alpha k+a k^{2}\right) U+(c-\lambda) k U^{3}+b U^{5}=0$,

Imaginary Part:

$\mathrm{v}+\alpha+2 \mathrm{ak}+(\mathrm{c}+3 \lambda+2 \mu) \mathrm{U}^{2}=0$

resulting in the two constraints

$\mathrm{v}=-\alpha-2 \mathrm{ak}$ and $\mathrm{c}+3 \lambda+2 \mu=0$.

Now, Eq. (12) gives the soliton speed $\mathrm{v}$ whereas integration of Eq. (10) will yield the soliton profile.

\section{Solving Eq. (10) by the first integral method}

As stated earlier, let us introduce the variables

$X(\xi)=U(\xi), Y(\xi)=U^{\prime}(\xi)$

Then, we have

$\left\{\begin{array}{c}Y(\xi)=X^{\prime}(\xi), \\ Y^{\prime}(\xi)=U^{\prime \prime}(\xi) \\ =\frac{\left(\omega+\alpha k+a^{2}\right)}{a} X-(c-\lambda) \frac{k}{a} X^{3}-\frac{b}{a} X^{5} .\end{array}\right.$

According to the first integral method, it is assumed that $X(\xi)$ and $\mathrm{Y}(\xi)$ are non-trivial solutions of Eq. (14) and the polynomial $\mathrm{Q}(\mathrm{X}, \mathrm{Y})=\sum_{\mathrm{j}=0}^{\mathrm{m}} \mathrm{a}_{\mathrm{j}}(\mathrm{X}) \mathrm{Y}^{\mathrm{j}}(\xi)$ is an irreducible polynomial in the complex domain $\mathrm{C}[\mathrm{X}, \mathrm{Y}]$ such that

$Q(X(\xi), Y(\xi))=\sum_{j=0}^{m} a_{j}(X) Y^{j}=0$

where $a_{j}(X)(j=0,1,2,3, \ldots m)$ are polynomials in $X$ and $\mathrm{a}_{\mathrm{m}}(\mathrm{X}) \neq 0$.

By division theorem, there exists a polynomial $[\mathrm{g}(\mathrm{X})+$ $\mathrm{h}(\mathrm{X}) \mathrm{Y}(\xi)]$ in the complex domain $\mathrm{C}[\mathrm{X}, \mathrm{Y}]$ such that 
$\frac{\mathrm{dQ}}{\mathrm{d} \xi}=\frac{\partial \mathrm{Q}}{\partial \mathrm{X}} \frac{\mathrm{dX}}{\mathrm{d} \xi}+\frac{\partial \mathrm{Q}}{\partial \mathrm{Y}} \frac{\mathrm{dY}}{\mathrm{d} \xi}$

$=\sum_{j=0}^{m} a_{j}^{\prime}(X) Y^{j+1}+\sum_{j=0}^{m} j a_{j}(X) Y^{j-1} Y^{\prime}$

$=[g(X)+h(X) Y] \sum_{j=0}^{m} a_{j}(X) Y^{j}$.

Case I: $m=1$ :

Substituting Eq. (14) in Eq. (16), we obtain

$a_{0}^{\prime}(X) Y+a_{1}^{\prime}(X) Y^{2}$

$+a_{1}(X)\left[\frac{\left(\omega+\alpha k+a k^{2}\right)}{a} X-(c-\lambda) \frac{k}{a} X^{3}-\frac{b}{a} X^{5}\right]$

$=[g(X)+h(X) Y]\left[a_{0}(X)+a_{1}(X) Y\right]$.

Equating the coefficients of $\mathrm{Y}^{\mathrm{j}}(\mathrm{j}=0,1,2)$ from both sides of Eq. (17), we obtain

$Y^{0}: g(X) a_{0}(X)$

$=a_{1}(X)\left[\frac{\left(\omega+\alpha k+a^{2}\right)}{a} X-(c-\lambda) \frac{k}{a} X^{3}-\frac{b}{a} X^{5}\right]$,

$Y^{1}: a_{0}^{\prime}(X)=g(X) a_{1}(X)+h(X) a_{0}(X)$,

$Y^{2}: a_{1}^{\prime}(X)=h(X) a_{1}(X)$

Since $\mathrm{a}_{\mathrm{j}}(\mathrm{j}=0,1)$ are assumed to be polynomials, Eq. (20) results in $\mathrm{a}_{1}(\mathrm{X})=$ constant and $\mathrm{h}(\mathrm{X})=0$.

For simplicity, we take $\mathrm{a}_{1}(\mathrm{X})=1$.

Now, from Eqs. (18) and (19), balancing of degrees of $\mathrm{a}_{0}(\mathrm{X})$ and $\mathrm{g}(\mathrm{X})$ results in

$\operatorname{deg}\left[\mathrm{a}_{0}(\mathrm{X})\right]=3$ and $\operatorname{deg}[\mathrm{g}(\mathrm{X})]=2$.

Thus, we assume that

$a_{0}(X)=A_{0}+A_{1} X+A_{2} X^{2}+A_{3} X^{3}$

and

$g(X)=A_{1}+2 A_{2} X+3 A_{3} X^{2}$

where the coefficients $A_{j}(j=0,1,2,3)$ are to be determined with $\mathrm{A}_{3} \neq 0$

Substituting Eqs. (21) and (22) in Eq.(18) and then equating the coefficients of $\mathrm{X}^{\mathrm{j}}(\mathrm{j}=0,1,2,3,4,5)$ we obtain

$\mathrm{X}^{0}: \mathrm{A}_{0} \mathrm{~A}_{1}=0$

$X^{1}: A_{1}^{2}+2 A_{0} A_{2}=\frac{\omega+\alpha k+a k^{2}}{a}$

$X^{2}: A_{1} A_{2}+A_{0} A_{3}=0$

$X^{3}: 4 A_{1} A_{3}+2 A_{2}^{2}=(\lambda-c) \frac{k}{a}$,

$X^{4}: A_{2} A_{3}=0$

$X^{5}: A_{3}^{2}=-\frac{b}{a}$.

Solving the above system of equations, we obtain

$A_{0}=0, A_{1}= \pm \sqrt{\frac{\omega+\alpha k+a k^{2}}{a}}$

$A_{2}=0, A_{3}= \pm \sqrt{-\frac{b}{3 a}}$
Substituting the above values of $A_{j}(j=0,1,2,3)$ in Eqs. (21) and (22), we obtain

$$
a_{0}(X)= \pm \sqrt{\left(\frac{\omega+\alpha k+a k^{2}}{a}\right)} X \pm \sqrt{-\frac{b}{3 a}} X^{3}
$$

and

$$
g(X)= \pm \sqrt{\frac{\omega+\alpha k+a k^{2}}{a}} X \pm \sqrt{-\frac{3 b}{a}} X^{2}
$$

Now, using Eqs. (13), (15) and (23), we write

$\mathrm{Y}=-\mathrm{a}_{0}$

Or, $\frac{d U}{d \xi}=\mp \sqrt{\frac{\omega+\alpha k+a k^{2}}{a}} U \mp \sqrt{-\frac{b}{3 a}} U^{3}$

which is a Bernoulli equation having the solution

$\mathrm{U}(\xi)= \pm\left[-\frac{3\left(\omega+\alpha \mathrm{k}+\mathrm{a} \mathrm{k}^{2}\right)}{\mathrm{b}\left\{1+\xi_{0} \mathrm{e}^{-2\left\{\sqrt{\left(\omega+\alpha \mathrm{k}+\mathrm{ak}^{2}\right) / \mathrm{a}}\right\} \xi}\right\}}\right]^{\frac{1}{2}}$

where $\xi_{0}$ is a constant.

Considering the positive roots, we write

I: for $\xi_{0}>0$,

$\mathrm{U}(\xi)=\sqrt{-\frac{3\left(\omega+\alpha \mathrm{k}+\mathrm{ak}^{2}\right)}{\mathrm{b}}}\left[\frac{1}{1+\mathrm{e}^{-2\left\{\frac{\left(\omega+\alpha \mathrm{k}+\mathrm{ak}^{2}\right) \xi}{\mathrm{a}}-\frac{\ln \xi_{0}}{2}\right\}}}\right]^{\frac{1}{2}}$

$=\sqrt{-\frac{3\left(\omega+\alpha \mathrm{k}+\mathrm{ak}^{2}\right)}{2 \mathrm{~b}}}\left[1+\tanh \left\{\left(\sqrt{\frac{\omega+\alpha \mathrm{k}+\mathrm{ak}^{2}}{\mathrm{a}}}\right) \xi-\frac{\ln \xi_{0}}{2}\right\}\right]^{\frac{1}{2}}$

II: for $\xi_{0}<0$,

$\mathrm{U}(\xi)$

$=\sqrt{-\frac{3\left(\omega+\alpha \mathrm{k}+\mathrm{ak}^{2}\right)}{2 \mathrm{~b}}}\left[1+\operatorname{coth}\left\{\left(\sqrt{\frac{\omega+\alpha \mathrm{k}+\mathrm{ak}^{2}}{\mathrm{a}}}\right) \xi-\frac{\ln \left(-\xi_{0}\right)}{2}\right\}\right]^{\frac{1}{2}}$

III: for $\xi_{0}=0$,

$\mathrm{U}(\xi)= \pm \sqrt{-\frac{3\left(\omega+\alpha \mathrm{k}+\mathrm{ak}^{2}\right)}{\mathrm{b}}}$

Substituting Eqs. (9) and (27) in Eq. (8), we write the solutions of Eq.(1) as in the following.

i) $\operatorname{For} \xi_{0}=1$ :

$q(x, t)$

$=\sqrt{-\frac{3\left(\omega+\alpha \mathrm{k}+\mathrm{ak}^{2}\right)}{2 \mathrm{~b}}} \times$

$\left[1+\tanh \left\{\left(\sqrt{\frac{\omega+\alpha \mathrm{k}+\mathrm{ak}^{2}}{\mathrm{a}}}\right)(\mathrm{x}-\mathrm{vt})\right\}\right]^{\frac{1}{2}} \mathrm{e}^{\mathrm{i}(-\mathrm{kx}+\omega \mathrm{t}+\theta)}$

(28 a)

ii) $\operatorname{For} \xi_{0}=-1$

$q(x, t)$ 


$$
\begin{aligned}
& =\sqrt{-\frac{3\left(\omega+\alpha \mathrm{k}+\mathrm{ak}^{2}\right)}{2 b}} \times \\
& {\left[1+\operatorname{coth}\left\{\left(\sqrt{\frac{\omega+\alpha \mathrm{k}+\mathrm{ak}^{2}}{a}}\right)(\mathrm{x}-\mathrm{vt})\right\}\right]^{\frac{1}{2}} \mathrm{e}^{\mathrm{i}(-\mathrm{kx}+\omega \mathrm{t}+\theta)} .}
\end{aligned}
$$

iii) $\operatorname{For} \xi_{0}=0$ :

$q(x, t)=\sqrt{-\frac{3\left(\omega+\alpha k+a k^{2}\right)}{b}} e^{i(-k x+\omega t+\theta)}$

Here, Eq. (28a) represents a dark soliton, Eq.(28b) represents a singular soliton and Eq.(28c) represents a plane wave solution. In similar manners, we can obtain other solutions with $\mathrm{m}=$ $2,3,4$ which are more complex. Calculations for $m \geq 5$ must be dropped out as algebraic equations with degrees greater than or equal to 5 are generally not solvable.

\section{Conclusion}

In this paper, the first integral method is successfully applied in finding exact solutions of perturbed Gerdjikov-Ivanov equation. The performance of this method is found to be effective and reliable. The method can be applied in finding exact solutions of many nonlinear evolution equations encountered in the studies of social dynamics, science and engineering. One advantage of the method is that it is applicable to both integrable as well as non-integrable systems.

\section{References}

[1] Biswas, A, Ekici,M, Sonmezoglu,A, Mazid, F. B., Triki, H., Zhou,Q, Moshokoa, S. P., Belic M (2018)., Optical soliton perturbation for Gerdjikov-Ivanov equation by extended trial equation method, Optik 158, 747-752. https://doi.org/10.1016/j.ijleo.2017.12.191.

[2] Biswas, A., Yildrim,Y., Yasar, E., Triki, H., Alshomrani, A. S. Moshokoa, S. P., Belic, M. (2018), Optical soliton perturbation with full nonlinearity for Gerdjikov-Ivanov equation by trial equation method, Optik 157, 1214-1218. https://doi.org/10.1016/j.ijleo.2017.12.099.

[3] Biswas, A., Yildrim, Y., Yasar, E., Zhou, Q., Alshomrani, A. S. Moshokoa, S. P., Belic, M. (2018), Solitons for perturbed Gerdjikov-Ivanov equation in optical fibers and PCF by extended Kudryashov method, Opt. Quant. Electron. 50:149. https://doi.org/10.1007/s11082-018-1417-0.

[4] Feng Z. S. (2002), the first integral method to study the BurgersKortweg-de-Vries equation, J. Phys. A 35(2), 343-349. https://doi.org/10.1088/0305-4470/35/2/312.

[5] Feng, Z. S. (2002), on explicit exact solutions to the compound Burgers Kortweg-de-Vries equation, Phys. Lett. A 293, 57-66. https://doi.org/10.1016/S0375-9601(01)00825-8.

[6] Feng, Z. S. (2008), travelling wave behavior for a generalized Fisher equation, Chaos, Solitons, Fract. 38, 481-488. https://doi.org/10.1016/j.chaos.2006.11.031.

[7] Feng, Z. S. and Wang, X. H. (2002), the first integral method to the two dimensional Burgers-KdV equation, Phys. Lett. A 308, 173-178. https://doi.org/10.1016/S0375-9601(03)00016-1.

[8] Taghizadeh, N., Mirzazadeh, M., Farahrooz, F. (2011), Exact solutions of the nonlinear Schrodinger equation by the first integral method, J. Math. Anal. Appl. 374, 549-553. https://doi.org/10.1016/j.jmaa.2010.08.050.

[9] Bekir,A. and Unsal, O. (2012), Analytical treatment of nonlinear evolution equations using the first integral method, Pramana $J$. Phys. 79, 3-17. https://doi.org/10.1007/s12043-012-0282-9. 